\title{
Association between adipose tissue thickness and fibroid shrinkage using ultrasound-guided high-intensity focused ultrasound
}

\author{
G C W Peiser, ${ }^{1}$ MB ChB; K Jacobs, ${ }^{2}$ MB ChB, FC Rad (SA), MMed (Rad Diag); \\ R T Spence, ${ }^{3} \mathrm{MB}$ ChB, MMed (Surg), MPhil, PhD, FCS (SA); R Setzen, ${ }^{4} \mathrm{MB}$ ChB
}

\author{
${ }^{1}$ Department of Radiology, Faculty of Health Sciences, University of the Witwatersrand, Charlotte Maxeke Johannesburg Academic Hospital, \\ Johannesburg, South Africa \\ ${ }^{2}$ Department of Radiology, Faculty of Health Sciences, University of the Witwatersrand, Chris Hani Baragwanath Academic Hospital, \\ Johannesburg, South Africa \\ ${ }^{3}$ Department of Surgery, Faculty of Medicine, Dalhousie University, Halifax, Canada \\ ${ }^{4}$ Department of Obstetrics and Gynaecology, Chris Hani Baragwanath Academic Hospital, Johannesburg, South Africa
}

Corresponding author: G CW Peiser(gcwpeiser@gmail.com)

\begin{abstract}
Background. Ultrasound-guided high-intensity focused ultrasound (USgHIFU) is used to noninvasively treat symptomatic uterine fibroids. The thickness of the adipose tissue that the HIFU beam travels through is thought to be associated with decreased treatment outcome, although the evidence is anecdotal.

Objectives. To determine whether the thickness of adipose tissue affects the percentage volume shrinkage of the uterine fibroid after treatment.

Method. A retrospective study was performed at Chris Hani Baragwanath Academic Hospital (CHBAH) on patients who were treated with USgHIFU for symptomatic uterine fibroids between 11 October 2015 and 11 April 2018. Magnetic resonance imaging (MRI) images were used to measure the adipose tissue thickness and the percentage volume shrinkage of the uterine fibroid. Linear regression was used to determine an association between adipose tissue thickness and the percentage volume shrinkage of the uterine fibroid after treatment.

Results. A total of 71 female patients who attended the CHBAH HIFU clinic were included in the study. Mean adipose tissue thickness was $17.49 \mathrm{~mm}$ (95\% confidence interval (CI) 14.86 - 19.73). Mean pre- and post-treatment volume of the fibroid was $176 \mathrm{~mL}$ (95\% CI 123.26 - 208.57) and $109 \mathrm{~mL}$ (95\% CI 71.75 - 141.73), respectively. The mean fibroid volume reduction was $44.68 \%(p<0.0001)$. We found no significant relationship between adipose tissue thickness and change in volume ratio of the fibroid.

Conclusion. It is unclear whether adipose thickness affects treatment outcome when symptomatic uterine fibroids are ablated using USgHIFU. This study found no correlation between adipose tissue thickness and uterine fibroid shrinkage after HIFU treatment. Our findings suggest that the exclusion criteria for USgHIFU treatment of uterine fibroids should be reviewed.

Keywords. fibroid; uterine fibroid; leiomyoma; HIFU; High frequency focussed ultrasound; USgHIFU; South Africa; adipose.
\end{abstract}

S Afr J Obstet Gynaecol 2020;27(3):94-98. https://doi.org/10.7196/SAJOG.2020.v27i3.2002

Uterine fibroids are common benign tumours in pre-menopausal women, and they cause a significant burden of disease. ${ }^{[1]}$ High-intensity focused ultrasound (HIFU) is a minimally invasive, nonsurgical option offered by interventional radiology to treat symptomatic uterine fibroids. This novel technology uses ultrasound waves that converge on a single central point, heat the uterine fibroid tissue to a temperature between $65-85^{\circ} \mathrm{C}$ and denature the cells. ${ }^{[1]}$ It does not require a skin incision or interventional endovascular therapy. HIFU waves are transmitted from a probe placed on a patient's lower abdomen in the midline, with the beam focused on the uterine fibroid that is intended for treatment. The heat that is generated from this focused ultrasound beam results in coagulative necrosis of the uterine fibroid parenchyma, which then results in fibroid shrinkage. ${ }^{[2]}$ Studies have reported that HIFU treatment can shrink fibroids by up to $50 \%$ over the $12-24$ months after treatment. ${ }^{[3,4]}$ Ultrasound-guided HIFU (USgHIFU) treatment is offered to patients with symptomatic fibroids at Chris Hani Baragwanath Academic Hospital (CHBAH). Symptomatic uterine fibroids defined by the department include bloating, pelvic pain, constipation, anaemia, infertility, and compression of the genitourinary system. ${ }^{[5]}$ As far as we can ascertain, this is the only health facility offering this treatment in southern Africa.
This is an important treatment option for women with symptomatic fibroids who wish to preserve their fertility. HIFU treatment of uterine fibroids was found to facilitate fertility, decrease the negative outcomes of pregnancy, and reduced pregnancy preparation time compared with other fibroid treatments. ${ }^{[6]}$ Preliminary results suggest that uterine fibroid ablation with HIFU is not associated with major risks during subsequent pregnancies, and is a noninvasive procedure, making this treatment option unique. ${ }^{[7]}$ Local studies have demonstrated that the HIFU treatment offered at $\mathrm{CHBAH}$ is effective and safe, ${ }^{[8]}$ although the treating clinician at $\mathrm{CHBAH}$ has offered an anecdotal opinion suggesting that there is variation in the success of treatment outcomes observed on follow-up visits. One possible factor that has been suggested to explain this variability is greater subcutaneous adipose tissue thickness, implying that adipose tissue may attenuate the HIFU beam and decrease its efficacy.

The primary objective of the present study was to determine whether the thickness of adipose tissue plays a role in the percentage shrinkage of uterine fibroids after treatment. The secondary objective was to ascertain whether the HIFU treatment offered at CHBAH is effective in the study population. 


\section{Methods}

\section{Participants}

Only patients who received USgHIFU therapy at CHBAH were considered for the present study. The departmental inclusion criteria of USgHIFU fibroid ablation therapy at $\mathrm{CHBAH}$ are:

- Pre-menopausal patients;

- Patients with symptomatic fibroids requiring treatment;

- The size of the uterus when measured clinically should be smaller than a gravid uterus of 16 weeks' gestation;

- The diameter of the uterine fibroid intended for treatment should be $>3 \mathrm{~cm}$ but $<15 \mathrm{~cm}$;

- Patients must be able to lie prone for a minimum of 1 hour and must be able to communicate with the treating clinician;

- Subcutaneous fat measurement of $<6 \mathrm{~cm}$; and

- The fibroid must not be pedunculated or intra-cavitatory. ${ }^{[8]}$

Patients who are being considered for HIFU treatment are first referred for a pelvic ultrasound. If the above criteria are met, the patients are then referred for an MRI of the pelvis (1.5 Tesla; General Electric, USA). The MRI images are used to measure the dimensions and exact location of the fibroid intended for treatment. Bladder or bowel in the line of the HIFU beam treatment plane is a contraindication for treatment as these structures could be damaged by the high-energy beam. Patients who meet the inclusion criteria are then given a date for USgHIFU treatment. All the patients undergo counselling prior to the treatment to ensure that the benefits and the risks of the procedure are understood. Informed consent is signed for the procedure as well as for conscious sedation in every patient.

CHBAH uses the JC200 focused ultrasound tumour therapeutic system (Chongqing Haifu Tech Company Ltd., China). During the procedure, the patient lies prone on the HIFU treatment bed with the abdomen submerged in a water bath. Air bubbles are carefully removed by hand from the patient's abdomen as these would scatter the ultrasound waves and weaken the beam. The HIFU beam probe is placed on the patent's abdomen directly anterior to the fibroid intended for treatment. Conscious sedation is administered (this is done by an anaesthetic registrar at $\mathrm{CHBAH}$ ). The ultrasound waves are directed towards a single central point within the fibroid to heat and destroy the targeted cells. These ablated cells result in uterine fibroid shrinkage, which is seen over the next 24 months. A HIFU treatment typically lasts between 1 and 3 hours, and multiple sessions may be needed to successfully ablate the targeted fibroid.

\section{Inclusion criteria}

A retrospective study was conducted on patients who had received USgHIFU fibroid ablation therapy between 11 October 2015 and 11 April 2018 at CHBAH. Only patients who received an MRI before the procedure and an MRI between 6 and 12 months after the procedure were included in the present study.

\section{Statistical analysis}

MRI images were used to determine the adipose thickness (in $\mathrm{mm}$ ) directly anterior to the treated fibroid on the pre-treatment MRI scan and change in volume between the pre- and post-treatment MRI scans. The software program R (version 3.5.1; R Studio, USA) was used for statistical analysis, and the results were plotted on scatter graphs. The patient data were anonymised, and the Protection of Personal Information (POPI) Act was adhered to. The primary outcome was to determine whether fibroid shrinkage had any statistical correlation with the thickness of the adipose tissue in the patients who were treated. The secondary outcome was to check whether the percentage shrinkage of the uterine fibroid treated with HIFU at CHBAH corresponded to the percentage shrinkage of uterine fibroids treated with HIFU at other centres internationally.

\section{Results}

A total of 71 patients met the inclusion criteria for the present study. All patients were of African descent, and their age ranged from 31 - 45 years. The patients who were selected for the present study received treatment between the dates of 11 October 2015 and 12 June 2017 (no treated patient met the inclusion criteria after the latter date). All patients received only one treatment session and had an MRI before the procedure and between 6 and 12 months after the procedure. The thinnest adipose tissue thickness measured was $3.8 \mathrm{~mm}$ and the thickest was $46.1 \mathrm{~mm}$. The mean adipose tissue thickness was $17.49 \mathrm{~mm}$ (95\% confidence interval (CI) 14.86 - 19.73). The mean pre-treatment volume of the fibroid was $176 \mathrm{~mL}$ (95\% CI 123.26 - 208.57) and the mean post-treatment volume of the fibroid $109 \mathrm{~mL}(95 \%$ CI 71.75 - 141.73). This is a mean reduction of $44.68 \%$ in size $(95 \%$ CI 38.21 - 51.14). The largest percentage volume shrinkage of a fibroid was $96 \%$ (Table 1 ).

A linear regression model demonstrates that the relationship between adipose tissue thickness and change in volume ratio is not significant $(p>0.05)$ (Fig. 1). A further generalised linear model was generated where the change in size was the response variable with both adipose tissue thickness and pre-treatment fibroid size included as predictor variables (an interaction term was included). It was found that even after accounting for pre-treatment fibroid size, the adipose thickness was insignificant $(p>0.05)$. To represent this visually, we created 4 graphs dividing the fibroid volumes in our study equally into quartiles by their pre-treatment volume (Fig. 2). There is a positive trend seen in the medium-small group, but this trend did not reach significance, suggesting that this trend may be an artifact. The thickness of the adipose tissue that the HIFU beam travels through is not a good predictor of the percentage volume shrinkage of the uterine fibroid. There is, however, a significant linear relationship between the pre-treatment volume and the percentage shrinkage of the uterine fibroid (Fig. 3). The correlation was determined with the input variable set as the pre-

Table 1. Summary of results

\begin{tabular}{|c|c|c|c|}
\hline & Min - $\max$ & Mean $(95 \% \mathrm{CI})$ & Range \\
\hline Adipose tissue thickness, $\mathrm{mm}$ & $3.8-46.10$ & $19.49(14.86-19.73)$ & 42.3 \\
\hline Pre-treatment volume, $\mathrm{mL}$ & $6.28-1040$ & $176.21(123.26-208.57)$ & 1033.72 \\
\hline Post-treatment volume, $\mathrm{mL}$ & $0.79-709.63$ & $109.20(71.75-141.73)$ & 708.84 \\
\hline Percentage shrinkage & $-2.44-96.91$ & $44.93(38.21-51.14)$ & 99.35 \\
\hline
\end{tabular}




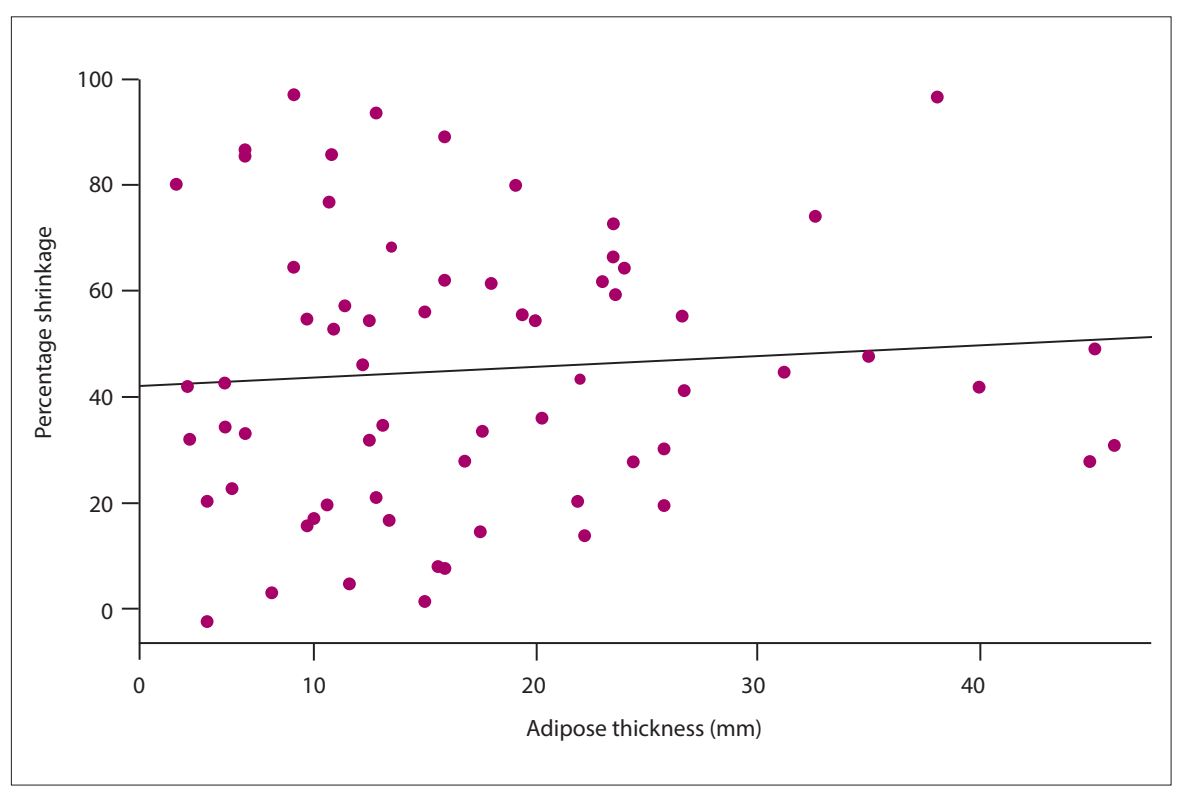

Fig. 1. Adipose thickness v. percentage shrinkage. A linear regression model demonstrating a statistically insignificant relationship between adipose tissue and percentage shrinkage of a uterine fibroid after HIFU treatment ( $\mathrm{p}>0.05)$. treatment volume of the uterine fibroid and the output variable the percentage shrinkage (Fig. 3). A statistically significant relationship was demonstrated between the size of uterine fibroids and the percentage volume shrinkage $(p<0.05)$. The larger uterine fibroids did not shrink as much as the smaller uterine fibroids after treatment, suggesting that the pre-treatment volume is important when predicting fibroid shrinkage.

\section{Discussion}

The primary goal of HIFU ablation of uterine fibroids is to reduce or alleviate the symptoms of uterine fibroids by shrinking their size ${ }^{[8]}$ A previous study at $\mathrm{CHBAH}$ assessing fibroid shrinkage, fibroid-related symptoms and quality-of-life assessments after HIFU treatment, successfully correlated a decrease in fibroid volume with reduced fibroid-related symptoms and an improved quality of life. ${ }^{[2]}$ It

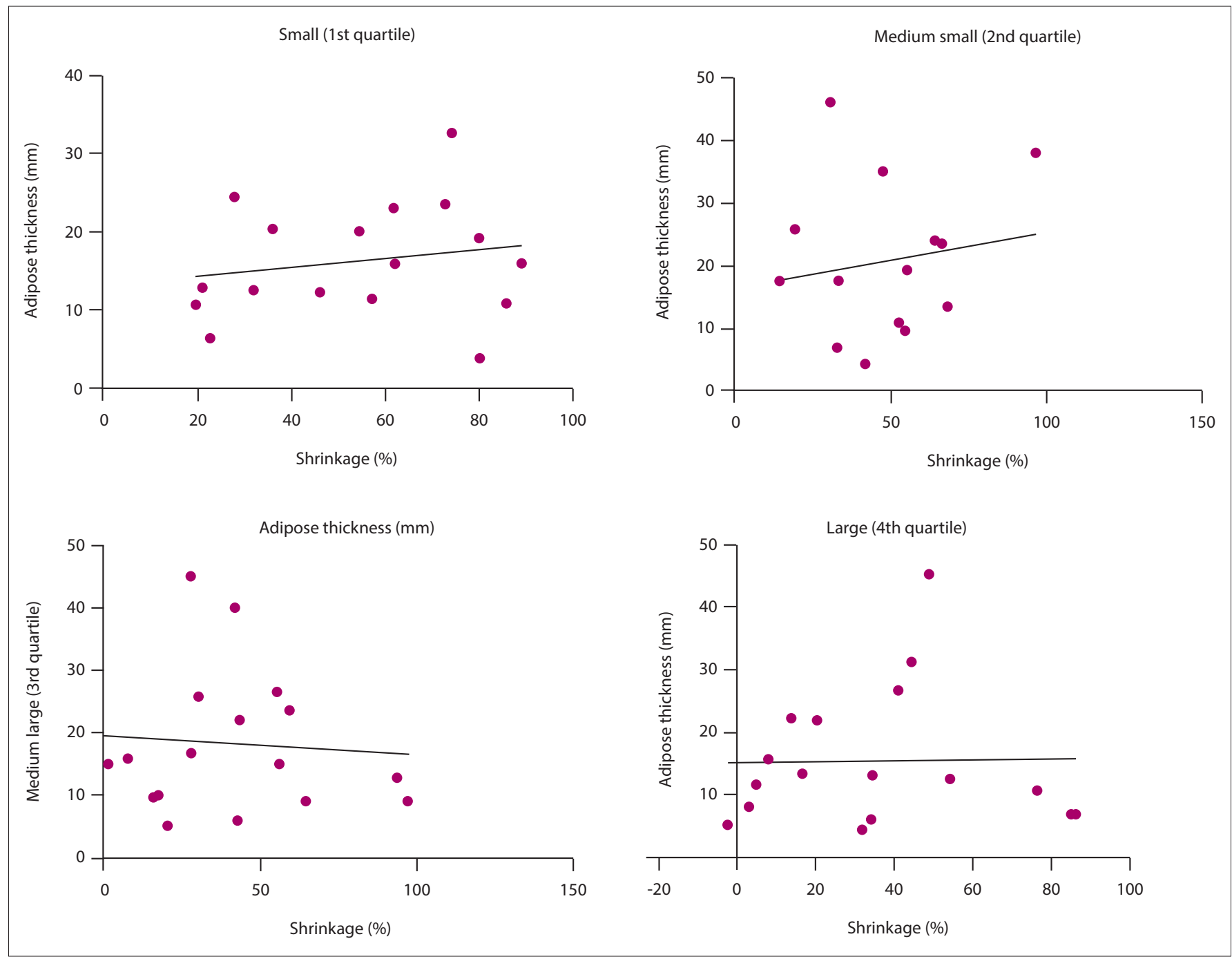

Fig. 2. Quartile graphs. In a generalised linear model where pre-treatment fibroid size was divided into quartiles and included as a predictor variable, depicted above, it was found that the adipose thickness was not an important predictor of the treatment outcome ( $>0.05)$. 


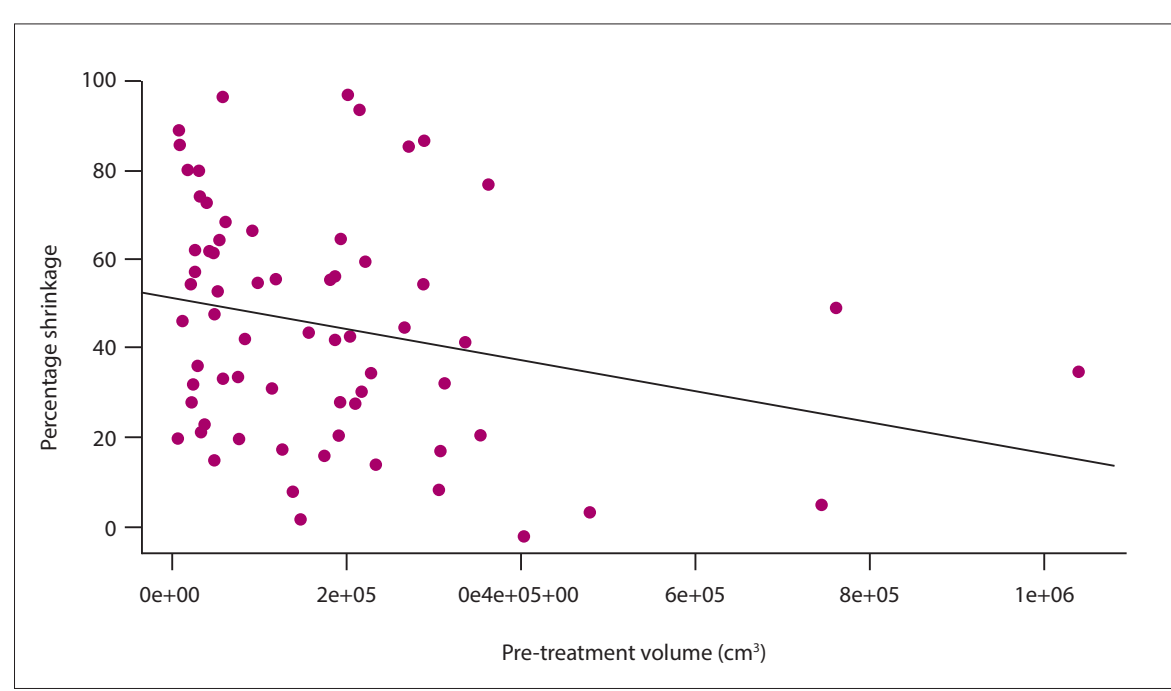

Fig. 3. Pre-treatment volume v. percentage shrinkage. This linear regression model depicts a significant linear relationship between the pre-treatment volume of the uterine fibroid and the percentage shrinkage the uterine fibroid undergoes after HIFU treatment.

can, therefore, be inferred that the percentage volume change ratio can be seen as an indication of the efficacy of the USgHIFU treatment, as smaller uterine fibroids cause fewer symptoms. The study population achieved an average shrinkage rate of $44 \%$ within 6 - 12 months following the HIFU treatment. This is in line with standard predicted outcomes, indicating that the treatment offered at $\mathrm{CHBAH}$ was successful. ${ }^{[2]}$ The percentage volume shrinkages seen in the present study would most likely result in symptomatic improvement although a qualitative questionnaire was not performed. Addressing the primary outcome, the present study determined that the thickness of adipose tissue that the HIFU beam travels through is not a good predictor of treatment outcome. There was a weak correlation between the adipose tissue thickness and the percentage volume shrinkage, but this relationship was not statistically significant. This is significant because an adipose tissue thickness $>6 \mathrm{~cm}$ is an exclusion criterion for HIFU treatment at CHBAH. This has been set in place because the chances of thermal damage to adipose tissue are increased with an increased adipose tissue thickness that the HIFU beam travels through. It is important to investigate the incidence of this side-effect and its correlation with fat thickness as it is not documented in the available literature. The present study suggests that the adipose tissue thickness does not affect the treatment outcome. Women who may benefit from this treatment but have adipose tissue thicknesses $>6 \mathrm{~cm}$ are not being offered this therapy as a treatment option at CHBAH. The reason for the adipose thickness exclusion criterion should be explored and potentially revised. This is important because patients with higher body mass indices are at higher risk of developing uterine fibroids. ${ }^{[9]}$ Furthermore, USgHIFU uterine fibroid ablation offers a valuable and unique treatment option for females of childbearing age as it allows them to preserve their fertility. ${ }^{[6]}$

The rationale for this exclusion criterion is that the energy of the HIFU beam is attenuated as it travels through the adipose tissue. There exists a proportional relationship between tissue thickness and beam scatter, with the potential for nontarget organ heating and tissue damage. Although adipose tissue thickness is a contributing factor, the distance of the probe to the fibroid being treated is more likely to predict whether patients will suffer side-effects. A fibroid situated anteriorly is much closer to the HIFU probe than a posteriorly located fibroid, so the chances of a patient suffering from sideeffects are lower in the case of the former. Unfortunately, the exclusion criterion of patients with an adipose thickness $>6 \mathrm{~cm}$ could contribute to bias in this present study, as we are unable to determine whether there would be a correlation between adipose tissue thickness and fibroid shrinkage in patients with adipose tissue thickness $>6 \mathrm{~cm}$.

A secondary outcome finding suggests that the uterine fibroid volume before the HIFU treatment is a better predictor of the percentage shrinkage after the treatment. The HIFU treatment is less effective on larger uterine fibroids. A possible explanation of this could be due to the HIFU ablation mechanism of action. The beam is focused on a single point and then moved in $3 \mathrm{D}$ space to cover the central volume of the uterine fibroid that is being ablated. A margin of $1 \mathrm{~cm}$ around the edges of the fibroid is left untreated to avoid damage to adjacent structures. This process is time-consuming and for larger uterine fibroids, it may not be practical or feasible to ablate the entire fibroid. The larger area could also result in fewer points being covered. Fibroids that were larger than a gravid uterus of 16 weeks' gestation were excluded from the study due to the standard manufacturer's criteria for treatment eligibility.

\section{Study limitations}

The study sample size was restricted by our inclusion criteria. A total of 236 patients who received treatment during the present study period were excluded because they either did not receive an MRI scan within the allocated timeframe after treatment or they did not receive an MRI scan after treatment at all. One of the two MRI scanners at the data collection site became dysfunctional during the allocated timeframe for retrospective data collection and the machine was not repaired. The dysfunctional MRI scanner, therefore, put a strain on the MRI department and decreased the number of MRI scans that the department was able to perform. As a result, the nonurgent MRI lists were restricted, and a decision was eventually made to stop MRI scans after treatment for women receiving USgHIFU fibroid ablation entirely. Our study is also limited by its retrospective nature. Although the treatment was shown to be effective in the majority of patients, it is unclear if this was as a result of USgHIFU treatment or due to the natural progression of uterine fibroids, as $7-40 \%$ of uterine fibroids may regress within 6 months to 3 years. ${ }^{[10]}$ Confounding factors were not excluded in our analysis, but this was a secondary outcome.

Although we found no association between adipose tissue thickness and fibroid volume shrinkage, we were not able to analyse the effect of HIFU in patients with an adipose thickness $>6 \mathrm{~cm}$. There may be a threshold thickness above which HIFU becomes ineffective, but we were not able to explore this further given the inherent exclusion criteria at CHBAH.

\section{Conclusion}

USgHIFU uterine fibroid ablation provides a valuable treatment option for women of childbearing age who want to preserve their fertility and improve other aspects of their 
wellbeing. Our results suggest that there is no clear relationship between the adipose tissue thickness and the percentage volume shrinkage of the treated uterine fibroid. This suggests that using adipose thickness as an exclusion criterion may result in patients who could benefit from this therapy being excluded from treatment unnecessarily. This, however, cannot be ascertained by this study as patients with adipose thickness $>6 \mathrm{~cm}$ were excluded.

\section{Recommendations}

This study suggests that there is no statistically significant relationship between the percentage volume shrinkage after HIFU treatment of a uterine fibroid and the adipose thickness $<6 \mathrm{~cm}$. A suggestion is that the distance between the centre of the uterine fibroid to be treated and the skin surface should be determined on pre-treatment MRI. The measurement should be set to review the maximum distance acceptable for HIFU treatment. The maximum range of a HIFU beam is $12 \mathrm{~cm}$. A study is suggested where the exclusion criterion of more than $12 \mathrm{~cm}$ should be used instead of an adipose thickness of greater than $6 \mathrm{~cm}$. This revision, which takes into account both the mechanism of the treatment and the potential side-effect of thermally damaged tissue, will assist by not excluding patients with an adipose thickness of $>6 \mathrm{~cm}$, who could benefit from this therapy.

The distance of the uterine fibroid from the ultrasound transducer is conceivably a better predictor of percentage fibroid shrinkage than adipose thickness. This is because the energy from the HIFU beam is attenuated by the tissues that it passes through. The uterine fibroid's location within the uterus (and therefore its distance to the anterior skin surface) will likely have a greater effect on the efficacy of treatment than the adipose thickness. A posteriorly located fibroid will receive less energy than an anteriorly located uterine fibroid. Further research is required to determine whether there is a relationship between the distance from the centre of the fibroid being treated to the cutaneous surface (where the HIFU transducer is situated) and the percentage shrinkage of the fibroid being treated. We also suggest further research to investigate other variables such as location of the fibroid, location of the uterus, the type of fibroid, whether there is a lower abdominal scar, dosimetry and the T2 characteristics of the fibroid to the treated, and comparing these variables using a multi regression analysis.

Declaration. This study was conducted in partial fulfilment of requirements for a MMed (Rad) degree.

Acknowledgements. We would like to thank Rheinhardt Scholtz and Brianna Beechler for their assistance with statistics.

Author contributions. GCWP and RS conceptualised the study. GCWP analysed and interpreted the data. KJ and RSP supervised the study and critically reviewed the draft manuscript. All the authors approved the final manuscript for publication.

Funding. None.

Conflicts of interest. None.

1. Rueff LE, Raman SS. Clinical and technical aspects of MR-guided high intensity focused ultrasound for treatment of symptomatic uterine fibroids. Sem Interven Rad 2013;30(4):347-353. https://doi org/10.1055\%2Fs-0033-1359728

2. Chen R, Keserci B, Bi H, et al. The safety and effectiveness of volumetric magnetic resonance-guided high-intensity focused ultrasound treatment of symptomatic uterine fibroids: Early clinical experience in China. J Therapeutic Ultrasound 2016;4:27. https://doi.org/10.1186\%2Fs40349-016-0072-9

3. Hindley J, Gedroyc WM, Regan L, et al. MRI guidance of focused ultrasound therapy of uterine fibroids: Early results. Am J Roentgenology 2004;183(6):1713-1719. https://doi. org/10.1055\%2Fs-0033-1359728

4. Hesley GK, Felmlee JP, Gebhart JB, et al. Noninvasive treatment of uterine fibroids: Early Mayo clinic experience with magnetic resonance imaging-guided focused ultrasound. Mayo Clinic Proceedings 2006;81(7):936-942. https://doi.org/10.4065\%2F81.7.936

5. He M, Jacobson H, Zhang C, Setzen R, Zhang L. A retrospective study of ultrasound-guided high intensity focused ultrasound ablation for multiple uterine fibroids in South Africa. Int J Hyperthermia 2018;34(8):1304-1310. https://doi.org/10.1080/02656736.2017.1421323

6. Keltz J, Levie M, Chudnoff S. Pregnancy outcomes after direct uterine myoma thermal ablation: Review of the literature. J Min Invasive Gynae 2017;24(4):538-545. https://doi.org/10.1016\%2Fj. bpobgyn.2008.04.002

7. Zou M, Chen L, Wu C, Hu C, Xiong Y. Pregnancy outcomes in patients with uterine fibroids treated with ultrasound-guided high-intensity focused ultrasound. Int J Obstet Gynae 2017;124(3):30-35 https://doi.org/10.1016\%2Fb978-1-4160-6160-1.00141-1

8. Zhang C, Jacobson H, Ngobese ZE, Setzen R. Efficacy and safety of ultrasound-guided high intensity focused ultrasound ablation of symptomatic uterine fibroids in Black women: A preliminary study. focused ultrasound ablation of symptomatic uterine fibroids in Black women: A prein
Int J Obstet Gynae 2017;124(3):12-17. https://doi.org/10.1111\%2F1471-0528.14738

9. Sato F, Nishi M, Kudo R, Miyake H. Body fat distribution and uterine leiomyomas. J Epi 1998;8(3):176-180. https://doi.org/10.2188/jea.8.176

10. Peddada SD, Laughlin SK, Miner K, et al. Growth of uterine leiomyomata among premenopausal black and white women. Proc Nat Aca Sci 2008;105(50):19887-19892. https://doi.org/10.3410\%2 Ff.723920028.793507538

Accepted 26 April 2021 